\title{
Análisis del estado psicofísico de los pacientes en hemodiálisis
}

\author{
Guillermo Pedreira Robles, Ana Vasco Gómez, Cristina Herrera Morales, Yaiza Martínez Delgado, Ernestina \\ Junyent Iglesias
}

Servicio de Nefrología. Parc de Salut Mar. Barcelona. España

\section{Resumen}

Introducción: Los pacientes en hemodiálisis deben someterse a tratamientos no curativos y altamente invasivos, implicando importantes cambios en el estilo, hábitos y calidad de vida.

Objetivo: Conocer el estado psíquico y físico de los pacientes en programa crónico de hemodiálisis y su opinión acerca de su experiencia personal en las sesiones de hemodiálisis.

Material y Método: Estudio observacional descriptivo de corte transversal. Se utilizaron diferentes escalas de valoración para conocer en profundidad el estado psico-físico de la muestra seleccionada.

Resultado: Inclusión de 35 pacientes, con una media de $67,43 \pm 14,82$ años y estancia mediana en hemodiálisis de 20 meses [P25 4; P75 62,5]. El 71,43\% presentaba algún grado de dependencia para las actividades básicas (Barthel) y el $85,71 \%$ para las actividades instrumentales (Lawton-Brody). El 71,43\% presentaba algún grado de ansiedad (Hamilton) y el $77,14 \%$ la probabilidad de padecer depresión (Escala Goldberg). El 54,29\% presentaba algún grado de deterioro cognitivo (Examen Minimental). El 88,57\% percibe afectación en su calidad de vida en el aspecto físico y el $51,43 \%$ a nivel mental (SF-12). La experiencia en hemodiálisis fue valorada por el grupo como Regular, Mala o Muy Mala por el 51,43\%, un 62,86\%

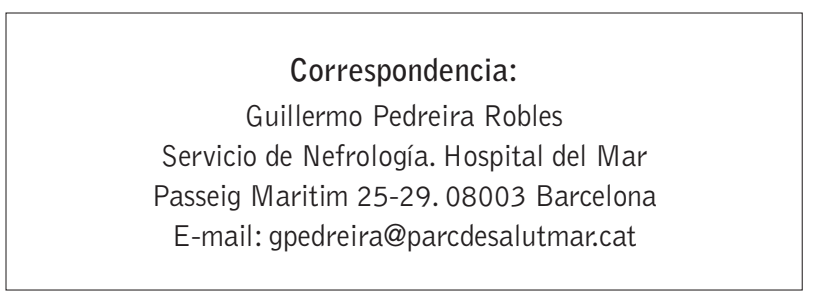

afirmó no hace nada durante las sesiones y un 57,14\% se siente atraído por la idea de participar en un Programa de Actividades Recreativas (según cuestionario de elaboración propia).

Discusión y Conclusiones: La mediana de los resultados en depresión, ansiedad, dependencia, deterioro cognitivo, percepción de calidad de vida y percepción del tratamiento está gravemente alterada. Según la bibliografía consultada, favorecer la realización de actividades lúdico-terapéuticas durante la hemodiálisis puede mejorar la actitud de los pacientes, ayudar a mantener un adecuado nivel de salud tanto físico como mental y mejorar la percepción del tratamiento.

PALABRAS CLAVE: hemodiálisis; depresión; ansiedad; dependencia; calidad de vida.

\section{Analysis of the psychophysical state of hemodialysis patients}

\begin{abstract}
Introduction: Hemodialysis patients should undergo non-curative and highly invasive treatments, involving significant changes in style, habits and quality of life.

Objective: To know the psychic and physical state of patients in a chronic hemodialysis program and their opinion about their personal experience in hemodialysis sessions.

Material and Method: A cross-sectional observational descriptive study was carried out. Different rating scales were used to know in depth the psycho-physical state of the selected sample.
\end{abstract}


Results: Thirty-five patients were included, with an average age of $67.43 \pm 14.82$ years and a median hemodialysis time of 20 months [P25 4; P75 62.5]. $71.43 \%$ had some degree of dependency for basic activities (Barthel) and $85.71 \%$ for instrumental activities (Lawton-Brody). $71.43 \%$ presented some degree of anxiety (Hamilton) and $77.14 \%$ the probability of suffering depression (Goldberg Scale). $54.29 \%$ had some degree of cognitive deterioration (Minimental Exam). 88.57\% perceive impairment in their quality of life in the physical aspect and $51.43 \%$ in the mental level (SF-12). The experience in hemodialysis was rated by the group as 'Regular', 'Bad' or 'Very Bad' by $51.43 \%$. A $62.86 \%$ reported doing nothing during the sessions and $57.14 \%$ are attracted by the idea of participating in a recreational program (according to a self-created questionnaire).

Discussion and Conclusions: The median of the results in depression, anxiety, dependence, cognitive deterioration, perception of quality of life and perception of the treatment is seriously altered. According to the consulted bibliography, favoring the performance of ludic-therapeutic activities during hemodialysis can improve the attitude of patients, helping maintain an adequate level of both physical and mental health and improving the perception of treatment.

KEYWORDS: hemodialysis; depression; anxiety; dependence; quality of life.

\section{Introducción}

La Enfermedad Renal Crónica (ERC) implica para el paciente una pérdida total de la capacidad funcional de los riñones y, por tanto, de la capacidad de excretar los productos de desecho y el excedente de líquido de la sangre a través de la orina. A consecuencia de esta pérdida de funcionalidad, que puede llegar a consecuencia de diferentes enfermedades o patologías, el paciente debe someterse a alguna de las Técnicas de Sustitución Renal (TSR) existentes: Hemodiálisis (HD), Diálisis Peritoneal (DP) o Trasplante Renal $(T R)^{1}$. El TR es la técnica que garantiza mejores resultados de supervivencia y calidad de vida, pero la media que el paciente permanece en lista de espera para recibir un riñón es de 2 años. Durante este tiempo de espera, el paciente deberá someterse a una de las técnicas dialíticas existentes ${ }^{2}$.
El proceso de la HD tal y como está establecido habitualmente (4 horas por 3 sesiones semanales) es esencial para la supervivencia del paciente, pues sin él, el paciente moriría. Es un proceso seguro en el que se garantizan los máximos beneficios para las personas que lo requieren, pero, a pesar de esto, se pueden presentar diferentes complicaciones o problemas que acompañan a la propia técnica ya sea por su diseño, la infraestructura, los requerimientos adicionales de régimen y cumplimiento terapéutico, interferencias en la vida cotidiana, etc. Estos problemas que se pueden derivar tienen diferentes gravedades $y$, por tanto, consecuencias para el bienestar y seguridad de la persona. Se pueden englobar en función de si el área de afectación es física (problemas nutricionales, metabólicos, vasculares, mecánicos, funcionales, etc.) 0 emocional (depresión, ansiedad, estrés, disfunción sexual, problemáticas sociales y familiares, restricciones en el uso del tiempo personal, miedos y temores, etc. $)^{3}$.

Las necesidades dialíticas del paciente renal están solventemente atendidas en nuestro país y se garantizan los tratamientos adecuados a todas las personas afectadas por la ERC. Los controles de seguimiento de estos enfermos están establecidos según los protocolos internos de cada centro y validados por las guías clínicas de la Sociedad Española de Nefrología. Sin embargo, se ha demostrado ampliamente que existe un porcentaje alto de pacientes afectado por problemas mentales (como la depresión y la ansiedad) que no reciben la atención adecuada ${ }^{4}$. Esto no es algo aislado en la población que estamos describiendo. En la población general, un $40 \%$ de los casos de depresión no reciben ningún tipo de tratamiento ${ }^{5}$, y es un trastorno que afecta a entre el $2 \%$ y el $4 \%$ de la población general, incrementándose hasta el 5\% - 10\% en los pacientes que acuden frecuentemente a las consultas de atención primaria y hasta el 10\% - 14\% para los pacientes hospitalizados ${ }^{6,7}$.

Otra variable que está siendo ampliamente estudiada en el campo de las patologías crónicas es la degeneración en las capacidades físicas y el aumento en el nivel de dependencia que ocurre en estas poblaciones ${ }^{8}$. De hecho, si entendemos al individuo como un ser biopsicosocial, espiritual y cultural nos daremos cuenta de que el diagnóstico de una enfermedad crónica que requiere una alta atención y dedicación, como es la ERC, no solamente produce un cambio en la situación vital del individuo, sino que el mismo diagnóstico afecta y cambia todas las esferas de este. 
De estas informaciones partió el objetivo principal de este estudio: conocer el estado psíquico y físico de los pacientes en programa crónico de hemodiálisis y su opinión acerca de su experiencia personal en las sesiones de hemodiálisis. Como objetivo secundario nos planteamos preguntar si existe interés en participar en algún tipo de actividad durante las sesiones o no.

\section{Material y Método}

\section{Tipo de estudio}

Estudio observacional, descriptivo y de corte transversal.

\section{Muestra}

Se propuso entrar en el estudio a todos los pacientes en HD de nuestro centro entre enero y febrero de 2016. Como criterios de inclusión se marcaron: tener ERC, estar incluido en el programa de HD por un tiempo prolongado de más de 1 mes, ser mayor de 18 años y firmar el consentimiento informado. Como criterios de exclusión se propuso: no aceptar participar en el estudio, condiciones cognitivas severas o barrera idiomática que impedían contestar escalas de valoración y un estado de salud comprometido.

\section{Recogida de datos}

La recogida de datos se realizó en las sesiones de HD por parte del personal de enfermería del equipo. Las escalas autoadministradas fueron contestadas por los pacientes durante las sesiones 0 en el domicilio particular y las heteroadministradas fueron respuestas a modo de entrevista.

Se recogieron datos sociodemográficos y clínicos para el análisis y comprensión de la muestra: sexo, edad, etiología de base, meses totales en HD desde el inicio de la técnica, tipo de acceso vascular, horas semanales de tratamiento, horas totales por sesión, inclusión en lista de espera para TR, uso de dispositivos para la movilización, uso de silla de ruedas, amputaciones y tratamiento antidepresivo o ansiolítico.

\section{Variables a estudio e instrumentos de medida Estado físico}

Escala Barthel: Mide la capacidad de una persona para realizar 10 Actividades Básicas de la Vida Diaria (ABVD) para determinar su grado de independencia. Los resultados se clasifican en: totalmente independiente (100 puntos), dependencia ligera (9199 puntos), dependencia moderada (61-90 puntos), dependencia severa (21-60 puntos) y dependencia total $(0-20 \text { puntos })^{9}$.

Escala Lawton y Brody: Valora las Actividades Instrumentales de la Vida Diaria (AIVD) que se definen como las actividades que comportan cierta complejidad de interacción con el medio social. Los 8 ítems a evaluar se expresan en las mismas categorías que en la escala anterior: independiente (8 puntos), dependencia ligera (6-7 puntos), dependencia moderada (4-5 puntos), dependencia severa (2-3 puntos) y dependencia total (0-1 puntos) ${ }^{10}$.

\section{Estado mental}

Escala Hamilton: Cuestionario utilizado para calificar la severidad de la ansiedad en una persona. Evalúa 14 criterios, que corresponden a 14 síntomas de ansiedad, desde 0 (nada) hasta 4 (muy severo). Se pueden obtener dos subescalas correspondientes a los síntomas de ansiedad psíquica y a los síntomas de ansiedad somática. En estas dos subescalas, los resultados se clasifican en: sin ansiedad (0-5 puntos), ansiedad moderada (6-14 puntos) y ansiedad mayor (15-28 puntos). Para el total de los 14 ítems sumados se puede obtener un resultado total que clasifica la ansiedad en su aspecto más general en: sin ansiedad (0-6 puntos), ansiedad leve (7-17 puntos), ansiedad moderada (18-24 puntos) y ansiedad grave (25-56 puntos) ${ }^{11}$.

Escala Goldberg: Determina el nivel, grado o probabilidad de padecer trastornos de depresión y/o ansiedad. Cada una de las subescalas consta de 4 ítems iniciales para despistar si es o no probable que exista un trastorno, y un segundo grupo de 5 ítems que se formulan sólo si se obtienen respuestas positivas a las preguntas anteriores ( 2 o más en la subescala de ansiedad, 1 o más en la subescala de depresión). Los resultados se interpretan con la afirmación (valores entre 4 y 9) o negación (valores entre 0 y 3) de la probabilidad de padecer el trastorno encuestado ${ }^{12}$.

Examen Minimental: Herramienta clínica extendida para determinar el estado cognitivo. La evaluación de los resultados transcurre entre la normalidad (2730 puntos), la sospecha patológica (25-26 puntos), el deterioro (12-24 puntos), la demencia (9-11 puntos) y la demencia grave (0-8 puntos) $)^{13}$.

\section{Calidad de Vida Relacionada con la Salud (CVRS)}

Cuestionario SF-12: La CVRS está basada en la percepción de la persona y en el impacto que tiene la 
enfermedad o tratamiento en su capacidad para vivir una vida satisfactoria. Se estima que, una vez analizados los datos, los resultados son negativos por debajo del valor 50 en una escala que llega hasta el $100^{14}$.

\section{Percepción del tratamiento}

Se diseñó un cuestionario ad hoc específico para este estudio. Para la elaboración de este, nos basamos en la necesidad de participar en formas de entretenimiento de Virginia Henderson y la validamos internamente en el servicio para su aplicación. Proponemos tres preguntas de respuesta cerrada:

- ¿Cómo tolera usted la sesión de hemodiálisis?

Muy bien, bien, regular, mal o muy mal.

- ¿Actualmente invierte el tiempo de hemodiálisis de alguna manera?

Si o no.

- Si no lo emplea de ninguna manera, ¿le gustaría participar o realizar alguna actividad?

Si o no.

\section{Análisis estadístico}

El análisis estadístico de los resultados se realizó mediante el programa Excel (Microsoft, 2106). Las variables numéricas se han descrito como números absolutos y porcentajes, los resultados de las escalas se expresan en mediana y cuartiles o media y desviación estándar.

\section{Aspectos éticos}

Este estudio se basa en los principios básicos que guían la atención y la investigación en el ámbito sociosanitario: principio de autonomía o de respeto a las personas, principio de beneficencia, principio de justicia y principio de no maleficencia, recogidas en el Código de ética de las enfermeras de Cataluña, la Declaración de Helsinki y la ley 15/1999 de Protección de Datos Personales.

\section{Resultados}

Fueron incluidos en el estudio 35 pacientes, 25 hombres y 10 mujeres. Las características sociodemográficas y clínicas están presentadas en la Tabla 1.

\section{Valoración del estado físico}

Mediante las escalas de valoración funcional, obtuvimos que un $71,43 \%(n=25)$ presentaba algún grado de dependencia para las ABVD según los resultados de la escala Barthel (5,72\% dependencia total, $28,57 \%$
Tabla 1. Características sociodemográficas y clínicas de la muestra.

\begin{tabular}{|c|c|}
\hline Edad media (DE) & $67,43(14,82)$ años \\
\hline Estancia mediana en HD (cuartiles) & 20 meses (P25 4; P75 62,5) \\
\hline Causa de ER (\%; n) & $\begin{array}{l}\text { No filiada }(37,14 \% ; 13) \\
\text { HTA }(22,86 \% ; 8) \\
\text { Glomerulares }(17,14 \% ; 6) \\
\text { Otras causas }(14,29 \% ; 5) \\
\text { Poliquísticas }(2,86 \% ; 1) \\
\text { Vasculares }(2,86 \% ; 1) \\
\text { DM }(2,86 \% ; 1)\end{array}$ \\
\hline Acceso vascular $(\% ; n)$ & $\begin{array}{l}\text { FAVn }(60 \% ; 21) \\
\text { FAVp }(22,86 \% ; 8) \\
\text { CVCp }(14,29 \% ; 5) \\
\text { CVCt }(2,86 \% ; 1)\end{array}$ \\
\hline Inclusión en lista de TR (\%; n) & $\begin{array}{l}\text { Si }(17,14 \% ; 6) \\
\text { No }(82,86 \% ; 31)\end{array}$ \\
\hline Horas de tratamiento/sesión $(\% ; n)$ & $\begin{array}{l}>4 \text { h/sesión }(5,72 \% ; 2) \\
4 \text { h/sesión }(68,57 \% ; 24) \\
<4 \text { h/sesión }(25,71 \% ; 9)\end{array}$ \\
\hline Dispositivos para la movilización (\%; n) & $\begin{array}{l}\text { Si }(54,29 \% ; 19) \\
\text { No }(45,71 \% ; 16)\end{array}$ \\
\hline Movilización en silla de ruedas $(\% ; n)$ & $\begin{array}{l}\text { Si }(28,57 \% ; 10) \\
\text { No }(71,43 \% ; 25)\end{array}$ \\
\hline Amputaciones en EEII $(\% ; \mathbf{n})$ & $\begin{array}{l}\text { Si }(11,43 \% ; 4) \\
\text { No }(88,57 ; 31)\end{array}$ \\
\hline Tratamiento antidepresivo $(\% ; \mathbf{n})$ & $\begin{array}{l}\text { Si }(14,29 \% ; 5) \\
\text { No }(85,71 \% ; 30)\end{array}$ \\
\hline Tratamiento ansiolítico (\%; n) & $\begin{array}{l}\text { Si }(45,71 \% ; 16) \\
\text { No }(54,29 \% ; 19)\end{array}$ \\
\hline
\end{tabular}

DE (Desviación Estándar), P (Percentil) ER (Enfermedad Renal), HTA (Hipertensión Arterial), DM (Diabetes Mellitus), FAVn (Fistula ArterioVenosa Nativa), FAVp (Fistula ArterioVenosa Protésica), CVCp (Catéter Venoso Central Permanente), CVCt (Catéter Venoso Central Temporal), TR (Trasplante Renal), EEII (Extremidades Inferiores).

dependencia severa, $34,29 \%$ dependencia moderada y $2,85 \%$ dependencia ligera). La mediana en esta escala fue de 80 [P25 50; P75 100] correspondiendo esto al grupo de dependencia moderada.

Un $85,71 \%$ (n=30) presentaba algún grado de dependencia para las AIVD $(17,14 \%$ dependencia total, $25,71 \%$ dependencia severa, $20 \%$ dependencia moderada y $22,86 \%$ dependencia ligera) siendo la mediana de 4 [P25 2; P75 7] y correspondiendo nuevamente al grupo de dependencia moderada (ver Figura 1).

\section{Valoración del estado mental}

Los datos obtenidos de la valoración del estado psíquico (ansiedad, depresión y cognición) mostraron que un $71,43 \% \quad(n=25)$ presentaba algún grado de ansiedad 


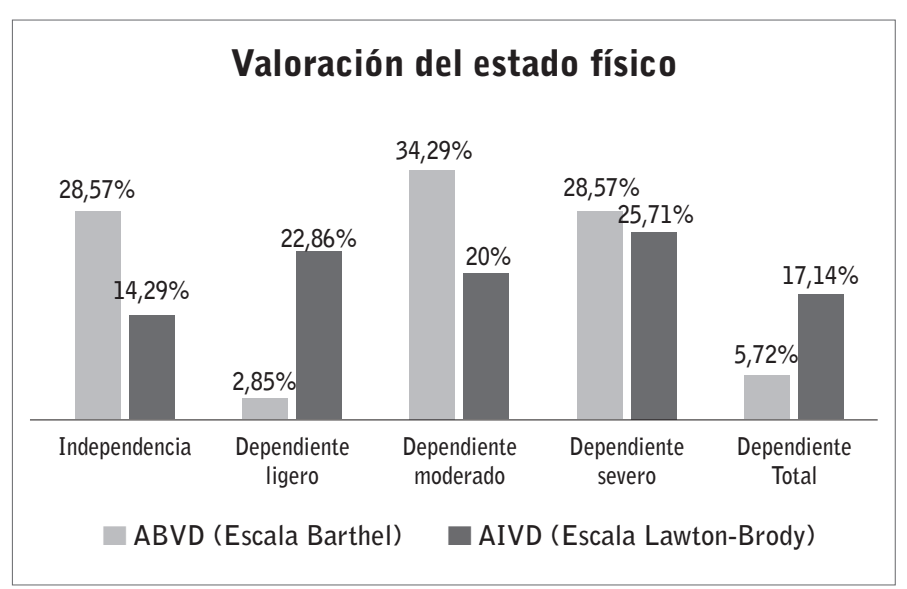

Figura 1. Valoración del estado físico.

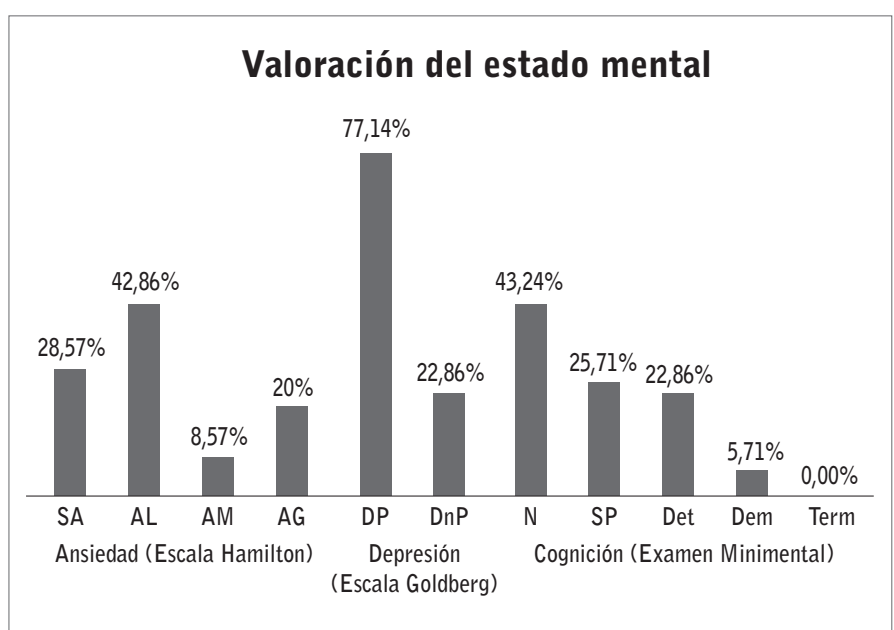

Figura 2. Valoración del estado mental.

SA (Sin Ansiedad), AL (Ansiedad Ligera), AM (Ansiedad Moderada), AG (Ansiedad Grave), DP (Depresión Probable), DnP (Depresión no Probable), N (Normalidad), SP (Sospecha Patologica), Det (Deterioro), Dem (Demencia), Term (Estado terminal).

según la Escala Hamilton de Ansiedad (20\% ansiedad grave, $8,57 \%$ ansiedad moderada y $42,86 \%$ ansiedad leve) (ver Figura 2). La mediana para esta escala fue de 10 [P25 6; P75 22], valor que corresponde a la categoría de ansiedad leve. En cuanto a los síntomas, un $11,42 \%$ padecían ansiedad mayor en la parte somática y un $17,14 \%$ ansiedad mayor en la parte psíquica.

En la parte de evaluada de depresión, el $77,14 \%$ $(n=27)$ presentaban una probabilidad de padecer este trastorno según la Escala Goldberg de Depresión (ver Figura 2) y la mediana obtenida para esta escala fue de 4 [P25 2; P75 6].

En cuanto a la evaluación del estado cognitivo, el Examen Minimental, reveló que un 43,24\% $(n=16)$ de los pacientes estaba dentro de la normalidad y el $54,29 \%$ $(n=19)$ restante presentaba algún tipo de deterioro cognitivo (un $25,71 \%$ se englobaba en el grupo de sospecha patológica, un $22,86 \%$ tenía un deterioro, un $5,71 \%$ estaba dentro del grupo de demencia) (ver Figura 2). La mediana para la escala de cognición y demencia fue de $26\left[\mathrm{P}_{25} 23 ; \mathrm{P}_{75} 29\right]$, clasificando al grupo en la categoría de sospecha patológica.

\section{Valoración de la calidad de vida relacionada con la salud}

La encuesta SF-12 determinó que, en el aspecto físico, un $88,57 \%(n=31)$ de los pacientes sintió afectación en su calidad de vida y, en el aspecto mental, un $51,43 \%(n=18)$ de los pacientes percibia afectación en su calidad de vida (ver Figura 3). Las medianas para las dos subescalas de CVRS fueron de 29,8 [P25 24,2; P75 38,9] para el aspecto físico y de 52,3 [P25 32,5; P75 58] para el aspecto mental.

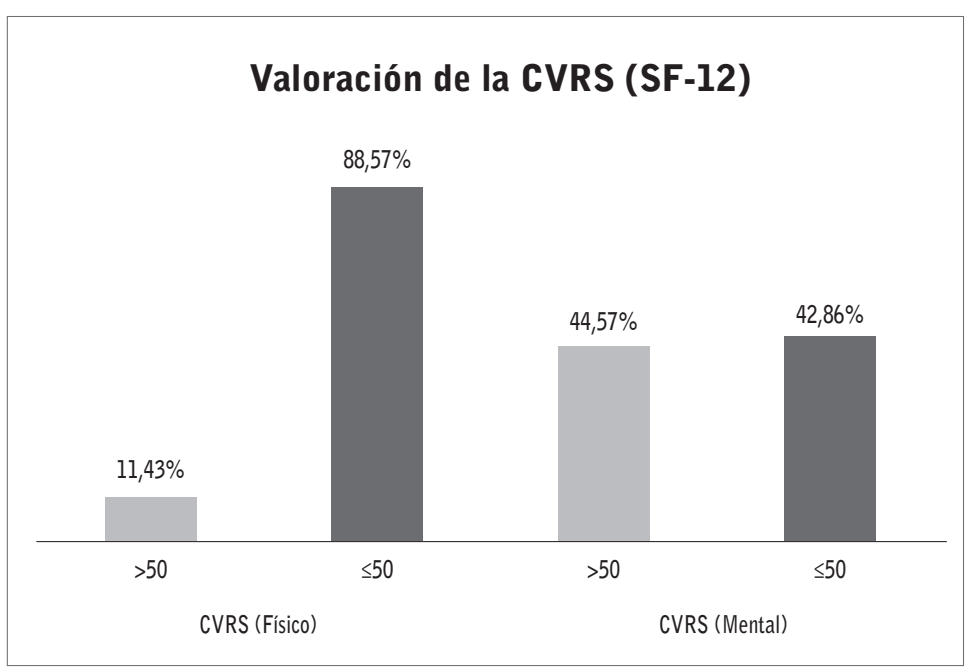

Figura 3. Valoración de la Calidad de Vida Relacionada con la Salud (CVRS).

\section{Valoración de la percepción del tratamiento}

La percepción personal de la tolerancia a la hemodiálisis fue valorada como muy buena por un $14,29 \%(n=5)$, un $34,29 \%$ la valoró como buena ( $n=12)$, un $22,86 \%$ comentó pasarlo regular $(n=8)$ frente a un $20 \%$ que valoró su experiencia como mala $(n=7)$ y un $8,57 \%$ $(n=3)$ que la calificó como muy mala (ver Figura 4). El $51,43 \%(n=18)$ de los pacientes valoró su experiencia en hemodiálisis como regular, mala o muy mala.

Un 62,86\% (n=22) afirmó no invertir el tiempo de las sesiones de hemodiálisis en ninguna actividad (ver Figura 4). 
Al $57,14 \%(n=20)$ le atraía la posibilidad de participar en alguna actividad intradiálisis y el $42,86 \% \quad(n=15)$ restante no le gustó la idea de participar en ninguna actividad durante las sesiones (ver Figura 4).

\section{Valoración de la percepción del tratamiento}

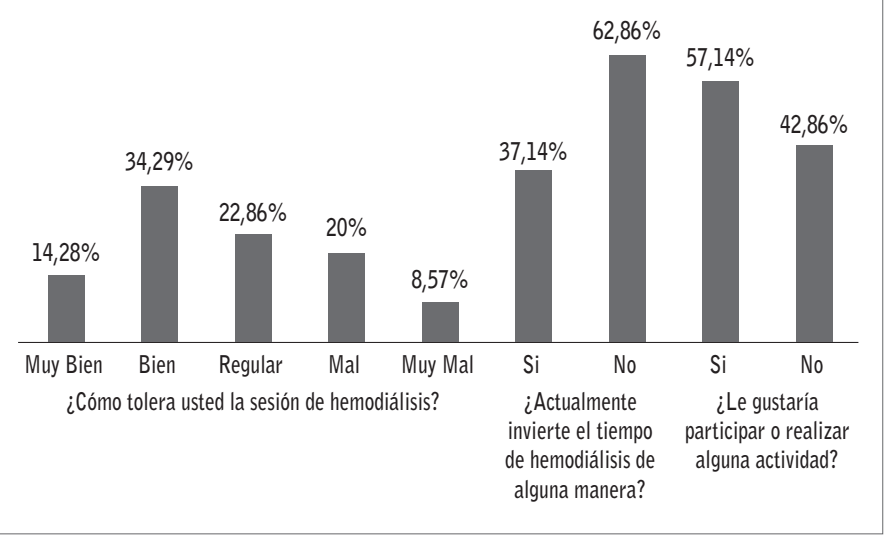

Figura 4. Valoración de la percepción del tratamiento.

\section{Discusión}

Tras analizar los datos obtenidos, encontramos un perfil de paciente con una edad media de 67,43 años, dependiente moderado, con trastornos de ansiedad leves-moderados y probabilidad alta de padecer trastornos depresivos, con percepciones de afectación en la calidad de vida relacionada con su salud en el aspecto mental, pero sobre todo en el aspecto físico y con alteraciones cognitivas leves. Derivado de estos datos, comprobamos que había apartados que presentaban alteraciones y que requieren de actuación por parte del equipo asistencial.

Centrándonos primero en la parte de dependencia, como hemos visto, un alto número de pacientes presentaban un grado moderado, severo o total de dependencia para las ABVD y las AIVD. De todos los pacientes, más de la mitad usaban un dispositivo de ayuda para la movilización y más de un cuarto de los pacientes examinados se movilizaban en silla de ruedas habitualmente y no pueden deambular de ninguna manera, debido, también en parte, por un considerable número de pacientes con amputaciones en extremidades inferiores por patología vascular.

Muchas publicaciones han trabajado el estado físico y la hemodiálisis, presentando resultados favorables de mejoría en la a fuerza muscular, capacidad funcional y preservación vascular ${ }^{15,16}$. Estos estudios han concluido en que la realización de ejercicio físico durante las sesiones de HD supone una mejoría importante en el estado físico de los pacientes 0 , al menos, no supone un empeoramiento tan rápido de este.

En los apartados de ansiedad y depresión, los resultados que se han presentado apuntan a que la mayoría de los pacientes se clasifican dentro de los grupos que presentan algún grado de malestar para estos trastornos. Estos porcentajes de pacientes presentan, por definición, respuestas desadaptativas que pueden llegar a ser patológicas. No concluyendo en un diagnóstico, si podemos afirmar que había una gran mayoría de pacientes que van acompañados de trastornos de ansiedad y depresión asociados.

Para comparar estos datos con los pacientes que estaban siendo tratados por estos trastornos, vemos que la ansiedad estaba siendo tratada de manera acorde con los resultados obtenidos, pero, en cambio, la depresión estaba infratratada según lo expuesto. Este es un hecho que se evidencia habitualmente en la población general en la que se ha descrito que un $40 \%$ de los trastornos de estas características no recibe ningún tipo de tratamiento $0^{6,7,17}$.

En el apartado de cognición, el hecho de medir el deterioro cognitivo de los pacientes nos ayuda a identificar dificultades a la hora de planificar intervenciones y planes de curas específicos. Hay estudios que han demostrado que, por causas de edad, pluripatología asociada o nivel de estudios logrado, el estado cognitivo se ve afectado, siendo la prevalencia para la población general mayor de 65 años del $10 \%$ y aumentándose con la edad ${ }^{19}$. En la muestra examinada en nuestro estudio, este trastorno se veía alterado 5 veces más que en la descripción otorgada por la literatura. Además, en diferentes referencias se ha relacionado nivel de cognición alterado y trastornos de depresión y/o ansiedad ${ }^{19}$.

Todos estos datos objetivados en los resultados de las escalas presentadas son sustentados con la percepción que los pacientes perciben acerca de su estado físico y mental. La calidad de vida relacionada con la salud, tanto en el aspecto físico como en el aspecto mental, es un marcador sobre los efectos funcionales de una enfermedad y su terapia sobre un paciente. Asimismo, se define el impacto de estos efectos en los niveles de bienestar subjetivo, satisfacción y autoestima ${ }^{20,21}$. Al observar alteraciones en un $88,57 \%$ (aspecto físico) y un $51,43 \%$ (aspecto mental), se comprobó que no solo hay carencias objetivables en los pacientes evaluados por las escalas, sino que estas carencias son percibidas por los pacientes de manera muy notable. 
Derivado del análisis de los datos presentados y de los beneficios expuestos en diferentes publicaciones $22,23,24,25$, nos hemos marcado como propósito instaurar un programa de actividades que acompañarán las sesiones de hemodiálisis y que trabajarán en las diferentes carencias de los pacientes (físicas y psicológicas). Esta propuesta queda validada por el $57,14 \%$ de pacientes que respondieron afirmativamente a la pregunta de si estarían interesados en participar en un Programa de Actividades intradiálisis y por el $62,86 \%$ de los pacientes que afirman no invertir el tiempo de diálisis en ninguna actividad con las repercusiones psicológicas y físicas que ello comporta.

\section{Conclusiones}

A raíz de los resultados obtenidos se aprecia que a pesar de que la edad media de los pacientes evaluados no es muy alta, hemos comprobado que la prevalencia de trastornos de ansiedad, depresión, dependencia o deterioro cognitivo es elevada en el grupo examinado. Además, la percepción que el paciente en hemodiálisis tiene en relación con su tratamiento es negativa y manifestaron no realizar ningún tipo de actividad durante este tiempo, pero sentirse atraídos por la posibilidad de poder hacerlo. Por ello, y con el objetivo principal de intentar mejorar el estado físico y psicológico y la percepción del tratamiento, proponemos instaurar un Programa de Actividades intradiálisis en nuestro centro.

Como se ha presentado, existen grupos de trabajo en el estado español que han iniciado diferentes actividades y han reportado resultados satisfactorios. La ocupabilidad intradiálisis será un reto que plantearse por los diferentes servicios de hemodiálisis después de los resultados obtenidos y presentados en el presente documento.

\section{Agradecimientos}

Los autores quieren agradecer el apoyo, dedicación y tiempo en la elaboración del presente estudio a Silvia Collado Nieto, Lola Andreu Periz y, en especial, a todas nuestras compañeras/eros y pacientes del servicio de Nefrología del Hospital del Mar de Barcelona.

\section{El autor declara que no hay conflicto de interés.}

Recibido: 12 septiembre 2017

Revisado: 20 septiembre 2017

Modificado: 28 septiembre 2017

Aceptado: 2 octubre 2017

\section{Bibliografía}

1. Martínez Castelao A, Bover Sanjuán J, Górriz Teruel $\mathrm{JL}$, Segura de la Morena J. Documento de consenso sobre la Enfermedad Renal Crónica. [Internet] 2012. [Consultado 30 enero 2016]. Disponible en: http:// www.senefro.org/modules/noticias/images/v._5.doc consenso_final_131212_copyl.pdf.

2. Pendse SS, Singh A, Zawada Jr E. Inicio de la diálisis. En: Daugirdas JT, Blake PG, Ing TS. Manual de diálisis. Barcelona: Wolters Kluwer; 2008. 14-21.

3. Cohen SD, Holder-Perkins H, Kimmel PL. Cuestiones psicosociales en pacientes con insufficiencies renal crónica terminal. En: Daugirdas JT, Blake PG, Ing TS. Manual de diálisis. Barcelona: Wolters Kluwer; 2008. 451-457.

4. Martínez del Pozo M, Martínez Camps E, Andrés Ribes E. ¿Por qué no diagnosticamos los niveles de Depresión, Ansiedad y Estrés en los pacientes en Hemodiálisis? CIN [Internet] 2007 - $4^{\circ}$ Congreso de Nefrología en Internet. [Consultado abril 2016]. Disponible en: http://cin2007.uninet.edu/en/trabajos/ fulltext/95.pdf.

5. La Vanguardia (Efe). Un $40 \%$ de la población padece ansiedad o depresión y sólo la mitad se trata. La Vanguardia. 2014. Disponible en: http://www.lavanguardia.com/vida/20140213/54400237421/un-40de-la-poblacion-padece-ansiedad-o-depresion-y-solola-mitad-se-trata.html.

6. Palmer $S$, Vecchio $M$, Craig JC, Tonelli $M$, Johnson DW, Nicolucci A et al. Prevalence of depression in chronic kidney disease: systematic review and meta-analysis of observational studies. Kidney International. 2013;84(1):179-191.

7. Hedayati SS, Bosworth HB, Briley LP, Sloane RJ, Pieper CF, Kimmel PL et al. Death or hospitalization of patients on chronic hemodialysis is associated with a physician-based diagnosis of depression. Kidney International. 2008;74:930-936. 
8. Rodríguez Díaz MT, Cruz-Quintana F, Pérez-Marfil MN. Dependencia funcional y bienestar en personas mayores institucionalizadas. Index Enferm. 2014;23(1-2):36-40.

9. Mahoney FI, Barthel D. Functional evaluation: The Barthel index. Maryland State Medical Journal; 1965;14:56-61.

10. Lawton MP, Brody EM. Assessment of older people: Self-maintaining and instrumental activities of daily living. Gerontologist, 1969;9:179-186.

11. Lobo A, Chamorro L, Luque A, Dal-Ré R, Badia X, Baró $E$. Validación de las versiones en español de la Montgomery-Asberg Depression Rating Scale y la Hamilton Anxiety Rating Scale para la evaluación de la depresión y de la ansiedad. Med Clin. 2002;118(13):493-499.

12. Goldberg DP, Bridges K, Duncan-Jones P, Grayson D. Detecting anxiety and depression in general medical settings. British Medical Journal. 1988;297:897-899.

13. Llamas-Velasco S, Llorente-Ayuso L, Contador I, Bermejo-Pareja F. Versiones en español del Minimental State Examination (MMSE). Cuestiones para su uso en la práctica clínica. Revista de Neurología. 2015;61(8):363-371.

14. Gandek B, Ware JE, Aaronson NK, Apolone G, Bjorner $J B$, Brazier JE et al. Cross-validation of item selection and scoring for the SF-12 Health Survey in nine countries: results from the IQOLA Project. International Quality of Life Assessment. Journal of Clinical Epidemiology. 1998;51(11):1171-1178.

15. Junque Jiménez $A$, Esteve Simón V, Iza Pinedo G, Tomás Bernabeú E, Luceño Soler I, Paz López 0 et at. Resultados de un programa de ejercicio físico combinado con electroestimulación neuromuscular en pacientes en hemodiálisis. Rev Soc Esp Enfer Nefrol. 2013,16(3):161-167.

16. Peña-Amaro P, García-López J, Zagalaz-Sánchez ML, Jimeno-Ucles R, Expósito-Rodríguez A. El ejercicio físico en pacientes en insuficiencia renal crónica terminal y programa de hemodiálisis. Revista Diálisis y Trasplante. 2009;30(4):127-132.
17. Martínez del Pozo M, Martínez Camps E, Andrés Ribes E. ¿Por qué no diagnosticamos los niveles de Depresión, Ansiedad y Estrés en los pacientes en Hemodiálisis? CIN [Internet] $2007-4^{\circ}$ Congreso de Nefrología en Internet. [Consultado abril 2016] Disponible en: http://cin2007.uninet.edu/en/trabajos/ fulltext/95.pdf.

18. Gil Nieto D, Perera Díaz M, Sevane Fernández L. Estado cognitivo del paciente de edad avanzada en programa de hemodiálisis. Rev Soc Esp Enfer Nefrol. 2010;13(1):30-35.

19. Latorre Postigo JM, Montañés Rodríguez J. Depresión en la vejez: evaluación, variables implicadas y relación con el deterioro cognitivo. Revista de Psicopatologla y Psicología Clínica. 1997;2(3):243-264.

20. Schmidt S, Vilagut G, Garin 0, Cunillera 0, Tresserras $R$. Brugulat $P$ et al. Normas de referencia para el Cuestionario de Salud SF-12 versión 2 basadas en población general de Cataluña. Med Clin (Barc). 2012;139(14):613-625.

21. Alfonso Uraúa M. Calidad de vida relacionada con la salud: Elementos conceptuales. Rev Med Chile. 2010;138:358-365.

22. Bauça Capellà MA, Guenoun Sanz M. ¿Cómo influye en los pacientes crónicos de hemodiálisis la organización de actividades de ocio durante sus sesiones? Rev Soc Esp Enfer Nefrol. 2012;15(1):57/62.

23. Guenon Sanz M, Bauçà Capellà MA. ¿Mejora la ansiedad y depresión de los pacientes crónicos en diálisis con la realización de actividades recreativas durante las sesiones? Rev Soc Esp Enfer Nefrol. 2012;15(1):97-101.

24. Verdes Moreiras MC, Fernández de Juan T. Aplicación de un programa de Musicoterapia en pacientes portadores de Insuficiencia Renal Crónica con Tratamiento de Hemodiálisis. Revista Cubana de Psicología. 1994;11(1):89-96.

25. Amador Coloma R, Pons Raventós E, Espinosa Calderón C. Depresión y Ansiedad en pacientes en hemodiálisis: La creatividad para combatirlas. Rev Soc Esp Enfer Nefrol. XXXIV Congreso Nacional de la SEDEN. 2009; 34.

Este artículo se distribuye bajo una Licencia Creative Commons Atribución-NoComercial 4.0 Internacional. https://creativecommons.org/licenses/by-nc/4.0/

\section{Open Access (c) () \&}

